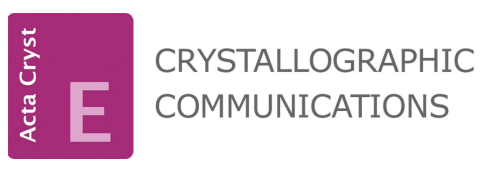

ISSN 2056-9890

Received 26 January 2021

Accepted 27 January 2021

Edited by W. T. A. Harrison, University of Aberdeen, Scotland

Keywords: crystal structure; dihydroquinolin2(1H)-one; piperazine; hydrochloride; molecular salt; dopamine $\mathrm{D}_{2}$ receptor; serotonin $5-\mathrm{HT}_{1}$ a receptor; hydrogen bonding; Hirshfeld surface analysis.

CCDC reference: 2059142

Supporting information: this article has supporting information at journals.iucr.org/e

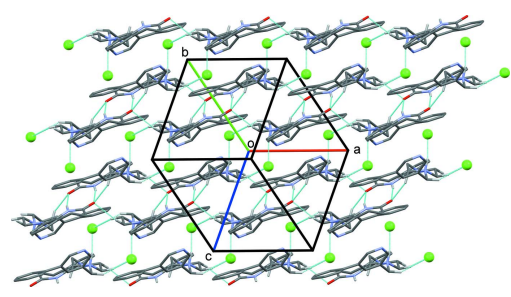

\section{Crystal structure and Hirshfeld surface analysis of the hydrochloride salt of 8-\{4-[(6-phenylpyridin-3- yl)methyl]piperazin-1-yl\}-3,4-dihydroquinolin- 2(1H)-one}

\author{
Nisar Ullah ${ }^{\mathrm{a}}$ and Helen Stoeckli-Evans ${ }^{\mathrm{b} *}$
}

${ }^{\mathbf{a}}$ Department of Chemistry, King Fahd University of Petroleum and Minerals, 31261 Dahran, Saudi Arabia, and ${ }^{\mathbf{b}}$ Institute of Physics, University of Neuchâtel, rue Emile-Argand 11, CH-2000 Neuchâtel, Switzerland. *Correspondence e-mail: helen.stoeckli-evans@unine.ch

The amine 8-\{4-[(6-phenylpyridin-3-yl)methyl]piperazin-1-yl\}-3,4-dihydroquinolin-2(1H)-one was crystallized as the hydrochloride salt, 4-(2-oxo-1,2,3,4tetrahydroquinolin-8-yl)-1-[(6-phenylpyridin-3-yl)methyl]piperazin-1-ium chloride, $\mathrm{C}_{25} \mathrm{H}_{27} \mathrm{~N}_{4}^{+} \cdot \mathrm{Cl}^{-}$(I.HCl). The conformation of the organic cation is halfmoon in shape enclosing the chloride anion. The piperidine ring of the 3,4dihydroquinolin-2 $(1 H)$-one moiety has a screw-boat conformation, while the piperazine ring has a chair conformation. In the biaryl group, the pyridine ring is inclined to the phenyl ring by 40.17 (7) and by $36.86(8)^{\circ}$ to the aromatic ring of the quinoline moiety. In the crystal, the cations are linked by pairwise $\mathrm{N}-\mathrm{H} \cdots \mathrm{O}$ hydrogen bonds, forming inversion dimers enclosing an $R_{2}^{2}(8)$ ring motif. The $\mathrm{Cl}^{-}$anion is linked to the cation by an $\mathrm{N}-\mathrm{H} \cdots \mathrm{Cl}$ hydrogen bond. These units are linked by a series of $\mathrm{C}-\mathrm{H} \cdots \mathrm{O}, \mathrm{C}-\mathrm{H} \cdots \mathrm{N}$ and $\mathrm{C}-\mathrm{H} \cdots \mathrm{Cl}$ hydrogen bonds, forming layers lying parallel to the $a b$ plane.

\section{Chemical context}

Schizophrenia is a psychiatric illness afflicting over $1 \%$ of the world's population. Adoprazine ${ }^{\odot}$ and Bifeprunox ${ }^{\odot}$ (Fig. 1) are two drugs that were developed for the treatment of Schizophrenia in the early 2000s. The main action of these two compounds is to combine dopamine $\mathrm{D}_{2}$ receptor blockade with serotonin $5-\mathrm{HT}_{1 \mathrm{~A}}$ receptor activation rather than antagonism (Feenstra et al., 2001, 2006). In continuing efforts in this field, Ullah and collaborators have synthesized a series of compounds that are structural analogues of Adoprazine ${ }^{\mathbb{C}}$ and Bifeprunox $^{(\mathcal{O}}$ (Ullah, 2012, 2014a,b; Ullah \& Al-Shaheri, 2012). These include a number of 1-aryl-4-(biarylmethylene)piperazines (Ullah, 2012), such as 8-\{4-[(6-phenylpyridin3-yl)methyl]piperazin-1-yl\}-3,4-dihydroquinolin-2(1H)-one (I), and 8-(4-\{[6-(4-fluorophenyl)pyridin-3-yl]methyl\}piperazin-1-yl)-3,4-dihydroquinolin-2(1H)-one (II) Ghani et al. (2014) have reported that the $\mathrm{D}_{2}$ receptor binding affinity of compounds I and II are $K_{\mathrm{i}}=28.4 \mathrm{n} M$ for I and $42.0 \mathrm{n} M$ for II. The $5-\mathrm{HT}_{1 \mathrm{~A}}$ receptor binding affinities were reported to be $K_{\mathrm{i}}$ $=4.30 \mathrm{n} M$ for $\mathbf{I}$ and $52.5 \mathrm{n} M$ for II. Hence, inserting a fluorine atom in the phenylpyridine unit in II did not improve its binding affinity compared to that of $\mathbf{I}$. Full details concerning these assays are given in Ghani et al. (2014).

The crystal structure of the hydrochloride salt of II has been reported previously (Ullah \& Altaf, 2014) and will be 
<smiles>Fc1ccc(-c2cncc(CN3CCN(c4cccc5c4OCCO5)CC3)c2)cc1</smiles>

adoprazine<smiles>O=C1CCc2cccc(N3CCN(Cc4cncc(-c5ccccc5)c4)CC3)c2N1</smiles>

Figure 1

Chemical diagrams for adoprazine, bifeprunoc and compounds I and II.

compared here to that of the hydrochloride salt of compound I.

\section{Structural commentary}

Due to the difficulty of forming suitable crystals for X-ray diffraction analysis compounds I and II were converted to their hydrochloride salts by treatment with $\mathrm{HCl}$ in $\mathrm{MeOH}$.<smiles>O=C1CCc2cccc(N3CC[NH+](Cc4ccc(-c5ccccc5)nc4)CC3)c2N1</smiles>

I. $\mathrm{HCl}$<smiles>O=C1CCc2cccc(N3CC[NH+](Cc4ccc(-c5ccc(F)cc5)nc4)CC3)c2N1</smiles>

II.HCI

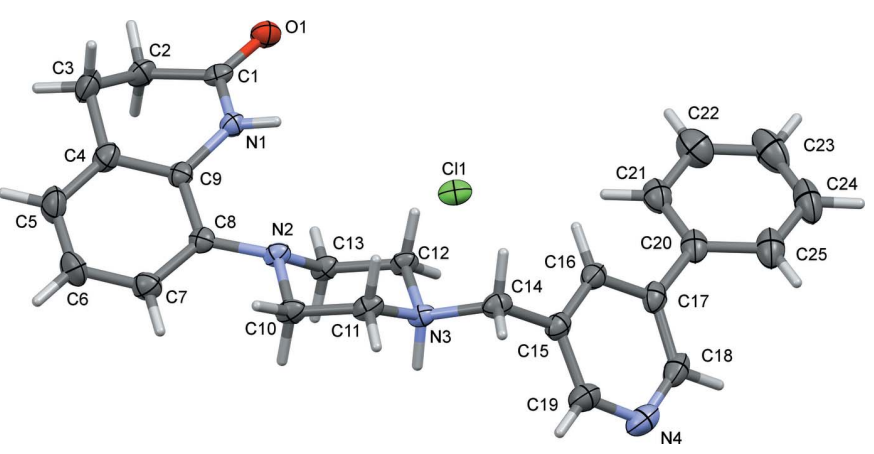

Figure 2

A view of the molecular structure of I.HCl, with atom labelling. The displacement ellipsoids are drawn at the $50 \%$ probability level.

The organic cation of I.HCl has a half-moon shape enclosing the chloride anion (Fig. 2). The molecular salt II.HCl crystallized as a monohydrate and here, while the cation also has a half-moon shape, it encloses the water molecule of crystallization (Ullah \& Altaf, 2014; see Fig. S1 in the supporting information). The two cations differ essentially in the conformation of the biaryl group (rings $B=\mathrm{N} 4 / \mathrm{C} 15-\mathrm{C} 19$ and $C=\mathrm{C} 20-\mathrm{C} 25)$ and their orientation with respect to the aromatic ring ( $A=\mathrm{C} 4-\mathrm{C} 9)$ of the 3,4-dihydroquinolin-2(1H)one moiety. This is illustrated by the view of their structural overlap, shown in Fig. 3. In I. HCl, pyridine ring $B$ is inclined to phenyl ring $C$ by $40.17(8)^{\circ}$ while in II.HCl the equivalent dihedral angle is $10.06(11)^{\circ}$. In $\mathbf{I} \cdot \mathbf{H C l}$, ring $A$ is inclined to rings $B$ and $C$ by 36.86 (8) and $14.16(8)^{\circ}$, respectively. These dihedral angles differ considerably from the dihedral angles in II.HCl, where $\operatorname{ring} A$ is inclined to rings $B$ and $C$ by 51.20 (9) and $41.40(11)^{\circ}$, respectively. In both compounds, the piperidine ring $(\mathrm{N} 1 / \mathrm{C} 1-\mathrm{C} 4 / \mathrm{C} 9)$ has a screw-boat conformation with the torsion angle $\mathrm{C} 1-\mathrm{C} 2-\mathrm{C} 3-\mathrm{C} 4$ being $-56.17(18)^{\circ}$ in I.HCl and $-55.6(2)^{\circ}$ in II.HCl. In both compounds, the piperazine ring $(\mathrm{N} 2 / \mathrm{N} 3 / \mathrm{C} 10-\mathrm{C} 13)$ has a chair conformation.

\section{Supramolecular features}

In the crystal of $\mathbf{I} \cdot \mathbf{H C l}$, the organic cations are linked by a pair of $\mathrm{N}-\mathrm{H} \cdots \mathrm{O}$ hydrogen bonds, forming an inversion dimer enclosing an $R_{2}^{2}(8)$ ring motif (Fig. 4 and Table 1). The $\mathrm{Cl}^{-}$ anion is linked to the cation by an $\mathrm{N}-\mathrm{H} \cdots \mathrm{Cl}$ hydrogen bond

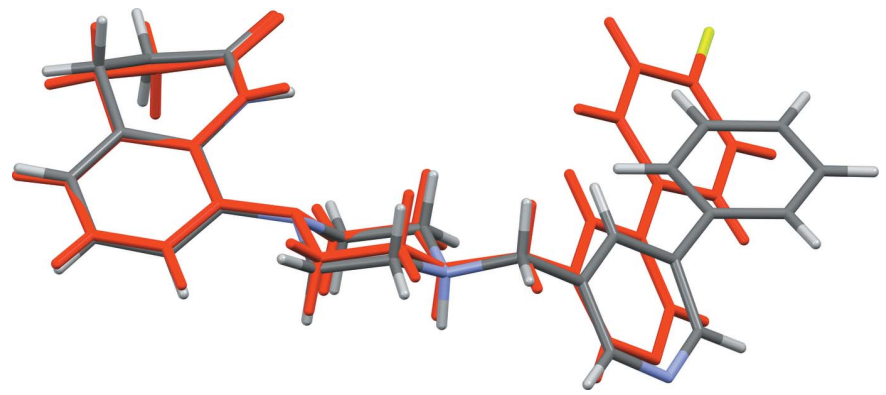

Figure 3

A view of the structural overlap of the cations of salts $\mathbf{I} \cdot \mathbf{H C l}$ and $\mathbf{I I} \cdot \mathbf{H C l}$; r.m.s. deviation $0.125 \AA$ (Mercury; Macrae et al., 2020). The structure of the II.HCl cation is given in red with the F atom in yellow (see also supplementary figure S1; Ullah \& Altaf, 2014). 
Table 1

Hydrogen-bond geometry $\left(\AA,^{\circ}\right)$.

$\mathrm{Cg}$ is the centroid of the C4-C9 ring.

\begin{tabular}{lllll}
\hline$D-\mathrm{H} \cdots A$ & $D-\mathrm{H}$ & $\mathrm{H} \cdots A$ & $D \cdots A$ & $D-\mathrm{H} \cdots A$ \\
\hline $\mathrm{N} 1-\mathrm{H} 1 N \cdots \mathrm{O} 1^{\mathrm{i}}$ & $0.85(2)$ & $2.01(2)$ & $2.844(2)$ & $168(2)$ \\
$\mathrm{N}^{\mathrm{ii}}-\mathrm{H} 3 N \cdots \mathrm{Cl} 1^{\mathrm{iii}}$ & $0.97(2)$ & $2.12(2)$ & $3.065(1)$ & $167(2)$ \\
$\mathrm{C} 10-\mathrm{H} 10 B \cdots \mathrm{O} 1^{\mathrm{iii}}$ & 0.99 & 2.40 & $3.151(2)$ & 132 \\
$\mathrm{C} 11-\mathrm{H} 11 A \cdots \mathrm{Cl} 1^{\mathrm{iii}}$ & 0.99 & 2.80 & $3.668(2)$ & 147 \\
$\mathrm{C} 12-\mathrm{H} 12 A \cdots \mathrm{Cl} 1$ & 0.99 & 2.81 & $3.520(2)$ & 129 \\
$\mathrm{C} 12-\mathrm{H} 12 B \cdots \mathrm{O} 1^{\mathrm{i}}$ & 0.99 & 2.26 & $3.123(2)$ & 144 \\
$\mathrm{C} 13-\mathrm{H} 13 A \cdots \mathrm{N} 1$ & 0.99 & 2.53 & $3.138(2)$ & 120 \\
$\mathrm{C} 14-\mathrm{H} 14 A \cdots \mathrm{Cl} 1^{\mathrm{iii}}$ & 0.99 & 2.71 & $3.585(2)$ & 147 \\
$\mathrm{C} 21-\mathrm{H} 21 \cdots \mathrm{Cl} 1$ & 0.95 & 2.83 & $3.757(2)$ & 165 \\
$\mathrm{C} 18-\mathrm{H} 18 \cdots C g^{\mathrm{ii}}$ & 0.95 & 2.83 & $3.487(2)$ & 127 \\
\hline
\end{tabular}

Symmetry codes: (i) $-x,-y+1,-z$; (ii) $-x+1,-y+2,-z$; (iii) $x+1, y, z$.

(Fig. 4 and Table 1). The dimers are linked by a $\mathrm{C}-\mathrm{H} \cdots \mathrm{O}$ hydrogen bond, forming ribbons propagating along the $a$-axis direction. The ribbons are then linked via $\mathrm{C}-\mathrm{H} \cdots \mathrm{Cl}$ hydrogen bonds to form layers lying parallel to the $a b$ plane (Fig. 5 and Table 1). There are $\mathrm{C}-\mathrm{H} \cdots \pi(\mathrm{C} 4-\mathrm{C} 9)$ contacts present within the layers (Table 1), but there are no significant contacts present between the layers.

In the crystal of II-HCI (Ullah \& Altaf, 2014; see Figs. S2 and S3, and Table S1 in the supporting information), the cations are linked by the water molecules of crystallization via $\mathrm{N}-\mathrm{H} \cdots \mathrm{O}_{\mathrm{w}}$ and $\mathrm{O}_{\mathrm{w}}-\mathrm{H} \cdots \mathrm{O}$ hydrogen bonds to form dimers with $R_{4}^{4}(12)$ ring motifs. The dimers are in turn linked by the $\mathrm{Cl}^{-}$anions, via $\mathrm{O}_{\mathrm{w}}-\mathrm{H} \cdots \mathrm{Cl} \cdots \mathrm{H}-\mathrm{N}$ hydrogen bonds, to form chains propagating along the $b$-axis direction. The chains are linked via $\mathrm{C}-\mathrm{H} \cdots \mathrm{Cl}$ and $\mathrm{C}-\mathrm{H} \cdots \mathrm{O}$ hydrogen bonds, forming layers parallel to the $a b$ plane.

In both cases, hydrogen-bonded layers are formed stacking along the $c$-axis direction and lying parallel to the $a b$ plane. There are no significant directional inter-layer contacts present in either crystal structure.

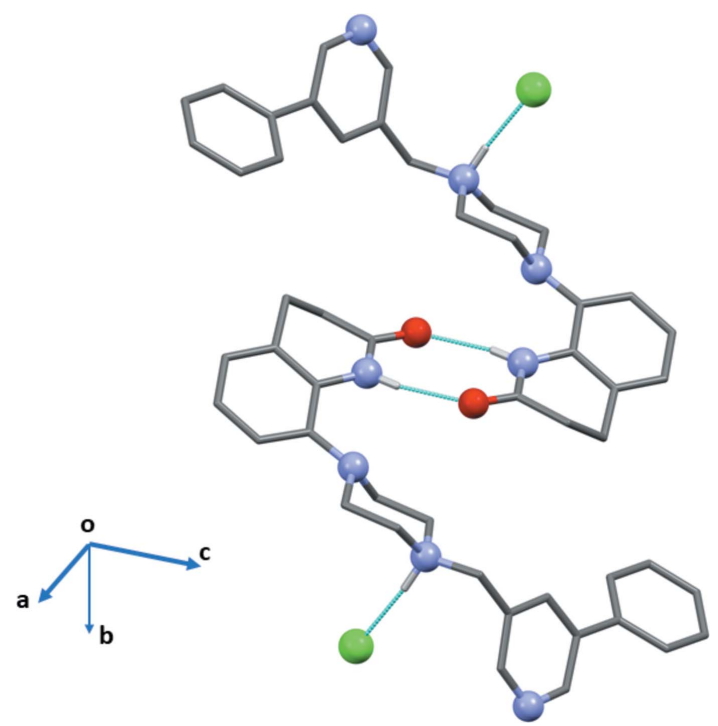

Figure 4

A view of the hydrogen bonded dimer formation in the crystal of salt I.HCl. Hydrogen bonds are shown as dashed lines (see Table 1).

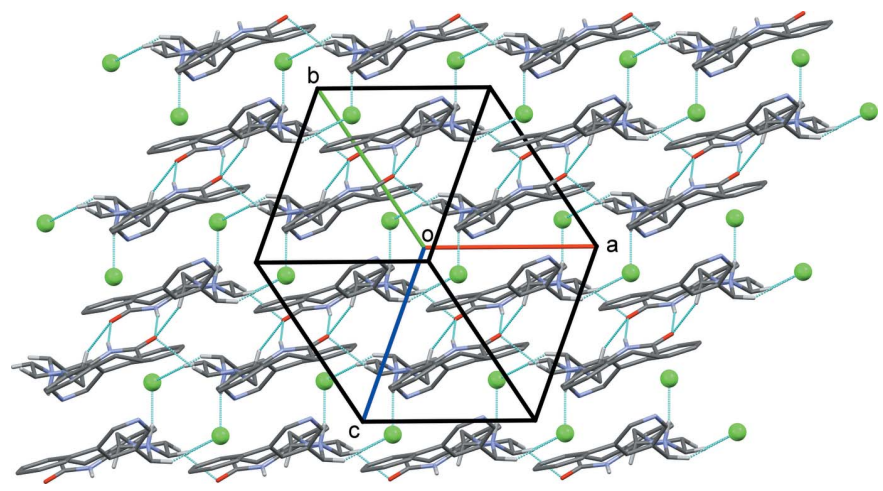

Figure 5

A view along the [111] direction of the crystal packing of salt I.HCl. Hydrogen bonds are shown as dashed lines (see Table 1).

\section{Hirshfeld surface analysis and two-dimensional fingerprint plots}

The Hirshfeld surface analysis (Spackman \& Jayatilaka, 2009) and the associated two-dimensional fingerprint plots (McKinnon et al., 2007) were performed with CrystalExplorer17 (Turner et al., 2017) following the protocol of Tiekink and collaborators (Tan et al., 2019).

The Hirshfeld surfaces are colour-mapped with the normalized contact distance, $d_{\text {norm, }}$ varying from red (distances shorter than the sum of the van der Waals radii) through white to blue (distances longer than the sum of the van der Waals radii). The Hirshfeld surfaces (HS) of I.HCl and II.HCl mapped over $d_{\text {norm }}$ are given in Fig. 6. It is evident from Fig. $6 a$ and $6 b$ that there are important contacts present in the crystals of both compounds, the strong hydrogen bonds (Table 1 and Table S1) being indicated by the large red zones.

The percentage contributions of inter-atomic contacts to the HS for both compounds are compared in Table 2. The twodimensional fingerprint plots for the title salt, I.HCl, and those for II.HCl, are compared in Figs. 7 and 8. They reveal, as expected, that the principal contributions to the overall HS surface involve $\mathrm{H} \cdots \mathrm{H}$ contacts at 51.5 and $42.1 \%$, respectively. The difference is attributed to the presence of $\mathrm{F} \cdots \mathrm{H} /$ $\mathrm{H} \cdots \mathrm{F}$ contacts in the crystal of $\mathbf{I I} \cdot \mathbf{H C l}$, amounting to $7.5 \%$. The second most important contribution to the HS is from the C. . H $/ \mathrm{H} \cdots \mathrm{C}$ contacts at 20.2 and $20.5 \%$, for $\mathbf{I} \cdot \mathbf{H C l}$ and

Table 2

Principal percentage contributions of inter-atomic contacts to the Hirshfeld surfaces of I.HCl and II.HCl..

\begin{tabular}{lll}
\hline Contact & $\begin{array}{l}\text { I.HCl } \\
\% \text { contribution }\end{array}$ & $\begin{array}{l}\text { II.HCl } \\
\% \text { contribution }\end{array}$ \\
\hline $\mathrm{H} \cdots \mathrm{H}$ & 51.5 & 42.1 \\
$\mathrm{C} \cdots \mathrm{H} / \mathrm{H} \cdots \mathrm{C}$ & 20.2 & 20.5 \\
$\mathrm{Cl} \cdots \mathrm{H} / \mathrm{H} \cdots \mathrm{Cl}$ & 10.1 & 12.8 \\
$\mathrm{O} \cdots \mathrm{H} / \mathrm{H} \cdots \mathrm{O}$ & 7.4 & 8.7 \\
$\mathrm{~N} \cdots \mathrm{H} / \mathrm{H} \cdots \mathrm{N}$ & 6.5 & 5.3 \\
$\mathrm{~F} \cdots \mathrm{H} / \mathrm{H} \cdots \mathrm{F}$ & - & 7.5 \\
$\mathrm{C} \cdots \mathrm{F} / \mathrm{F} \cdots \mathrm{C}$ & - & 1.4 \\
$\mathrm{C} \cdots \mathrm{C}$ & 2.9 & 0.8 \\
\hline
\end{tabular}




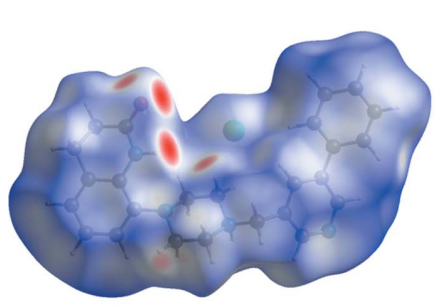

(a)

I. $\mathrm{HCl}$

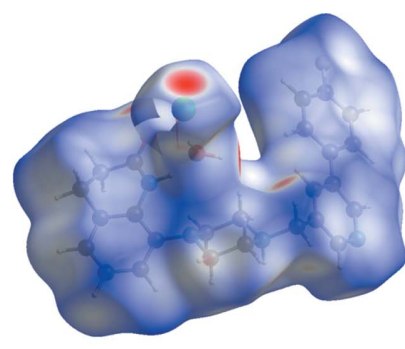

(b)

II. $\mathrm{HCl}$

Figure 6

The Hirshfeld surfaces of compounds I.HCl and II.HCl, mapped over $d_{\text {norm }}$ in the colour ranges of -0.5847 to 1.5642 au. and -0.5555 to 1.5111 au., respectively.

II.HCl, respectively. These are followed by the $\mathrm{Cl} \cdots \mathrm{H} \cdots \mathrm{Cl}$ contacts at 10.1 and $12.8 \%$ for I.HCl and II.HCl, respectively, and $\mathrm{O} \cdots \mathrm{H} / \mathrm{H} \cdots \mathrm{O}$ contacts at, respectively, 7.4 and $8.7 \%$. The $\mathrm{N} \cdots \mathrm{H} / \mathrm{H} \cdots \mathrm{N}$ contacts contribute, respectively, 6.5 and $5.3 \%$. The $\mathrm{C} \cdots \mathrm{C}$ contacts in $\mathbf{I} \cdot \mathbf{H C l}$ contribute $2.9 \%$, while the $\mathrm{C} \cdots \mathrm{F}$ contacts in II.HCl contribute $1.4 \%$. All other atom $\cdots$ atom contacts contribute $<1 \%$ to the HS for both compounds.

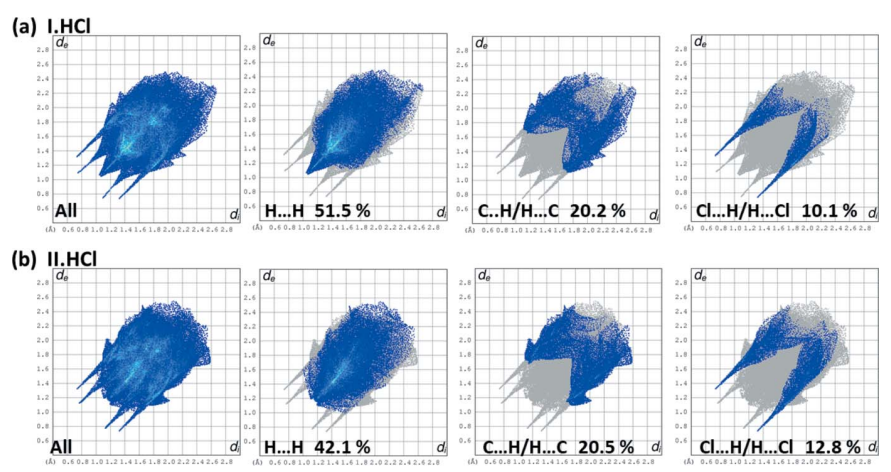

Figure 7

The two-dimensional fingerprint plots for compounds $(a)$ I.HCl and $(b)$ II.HCl, and those delineated into $\mathrm{H} \cdots \mathrm{H}, \mathrm{C} \cdots \mathrm{H} / \mathrm{H} \cdots \mathrm{C}$, and $\mathrm{Cl} \cdots \mathrm{H} /$ $\mathrm{H} \cdot \mathrm{Cl}$ contacts.

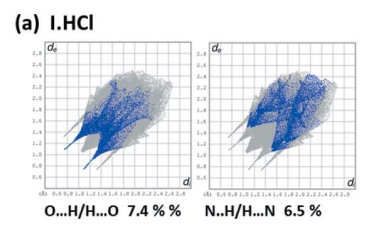

(b) $1 \mathrm{I.HCl}$
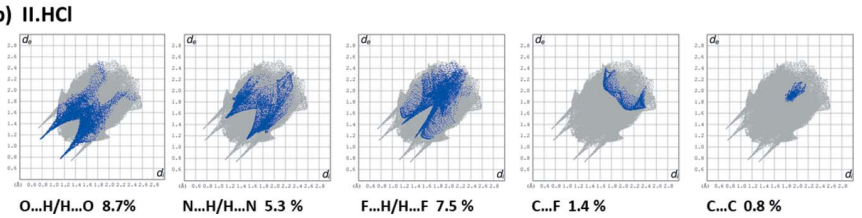

Figure 8

The two-dimensional fingerprint plots for compounds (a) I.HCl delineated into $\mathrm{O} \cdots \mathrm{H} / \mathrm{H} \cdots \mathrm{O}, \mathrm{N} \cdots \mathrm{H} / \mathrm{H} \cdots \mathrm{N}$ and $\mathrm{C} \cdots \mathrm{C}$, and $(b)$ II.HCl delineated into $\mathrm{O} \cdots \mathrm{H} / \mathrm{H} \cdots \mathrm{O}, \mathrm{N} \cdots \mathrm{H} / \mathrm{H} \cdots \mathrm{N}, \mathrm{F} \cdots \mathrm{H} / \mathrm{H} \cdots \mathrm{F}, \mathrm{C} \cdots \mathrm{F} / \mathrm{F} \cdots \mathrm{C}$ and $\mathrm{C} \cdot \mathrm{C}$ contacts.

\section{Database survey}

A search of the Cambridge Structural Database (CSD, Version 5.42, last update November 2020; Groom et al., 2016) for 8-(piperazin-1-yl)-3,4-dihydroquinolin-2(1H)-ones gave three hits for compounds $8-\left\{1-\left[\left(4^{\prime}\right.\right.\right.$-fluoro-[1, $1^{\prime}$-biphenyl $]-3$ yl)methyl]piperidin-4-yl\}-3,4-dihydroquinolin-2(1H)-one (III), that crystallized as a chloroform 0.25-solvate (CSD refcode FITSEI; Ullah \& Stoeckli-Evans, 2014), 8-[4-([1,1'biphenyl]-3-ylmethyl)piperazin-1-yl]quinolin-2(1H)-one (IV) (REYHIP; Ullah et al., 2017) and 8-[1-([1,1'-biphenyl]-3ylmethyl)piperidin-4-yl]-3,4-dihydroquinolin-2(1H)-one (V) (REYHEL; Ullah et al., 2017). Their chemical structures are shown in Fig. 9, together with those for compounds 8-[4-([1,1'biphenyl]-3-ylmethyl)piperazin-1-yl]-2-methoxyquinoline (VI) (AKUXIQ; Ullah \& Altaf, 2014), 8-(1-\{[5-(cyclopent-1en-1-yl)pyridin-3-yl]methyl\}piperidin-4-yl)-3,4-dihydroquinolin-2(1H)-one (VII) (AKUWOV; Ullah et al., 2015) and 8-\{1[3-(cyclopent-1-en-1-yl)benzyl]piperidin-4-yl\}-3,4-dihydroquinolin-2(1H)-one (VIII) (AKUWUB; Ullah et al., 2015).<smiles>O=C1CCc2cccc(C3CCN(Cc4cccc(-c5ccc(F)cc5)c4)CC3)c2N1</smiles><smiles>O=c1ccc2cccc(N3CCN(Cc4cccc(-c5ccccc5)c4)CC3)c2[nH]1</smiles>

III

IV<smiles>O=C1CCc2cccc(C3CCN(Cc4cccc(-c5ccccc5)c4)CC3)c2N1</smiles>

V<smiles>COc1ccc2cccc(N3CCN(Cc4cccc(-c5ccccc5)c4)CC3)c2n1</smiles>

VI<smiles>O=C1CCc2cccc(C3CCN(Cc4cncc(C5=CCCC5)c4)CC3)c2N1</smiles>

VII<smiles>O=C1CCc2cccc(C3CCN(Cc4cccc(C5=CCCC5)c4)CC3)c2N1</smiles>

VIII

Figure 9

Chemical diagrams of similar compounds deposited with the CSD (Groom et al., 2016); see $§ 5$ Database survey. 
Table 3

Experimental details.

\begin{tabular}{|c|c|}
\hline \multicolumn{2}{|l|}{ Crystal data } \\
\hline Chemical formula & $\mathrm{C}_{25} \mathrm{H}_{27} \mathrm{~N}_{4} \mathrm{O}^{+} \cdot \mathrm{Cl}^{-}$ \\
\hline$M_{\mathrm{r}}$ & 434.95 \\
\hline Crystal system, space group & Triclinic, $P \overline{1}$ \\
\hline Temperature $(\mathrm{K})$ & 173 \\
\hline$a, b, c(\AA)$ & $\begin{array}{l}8.4791(8), 10.4091(10) \\
\quad 13.6862(14)\end{array}$ \\
\hline$\alpha, \beta, \gamma\left({ }^{\circ}\right)$ & $90.138(8), 94.833(8), 113.745$ (7) \\
\hline$V\left(\AA^{3}\right)$ & $1100.88(19)$ \\
\hline$Z$ & 2 \\
\hline Radiation type & Мо $K \alpha$ \\
\hline$\mu\left(\mathrm{mm}^{-1}\right)$ & 0.20 \\
\hline Crystal size (mm) & $0.45 \times 0.33 \times 0.18$ \\
\hline \multicolumn{2}{|l|}{ Data collection } \\
\hline Diffractometer & Stoe $I P D S 2$ \\
\hline Absorption correction & Multi-scan (MULABS; Spek, 2020) \\
\hline$T_{\min }, T_{\max }$ & $0.379,1.000$ \\
\hline $\begin{array}{l}\text { No. of measured, independent and } \\
\text { observed }[I>2 \sigma(I)] \text { reflections }\end{array}$ & 13410, 4156, 2912 \\
\hline$R_{\text {int }}$ & 0.077 \\
\hline$(\sin \theta / \lambda)_{\max }\left(\AA^{-1}\right)$ & 0.609 \\
\hline \multicolumn{2}{|l|}{ Refinement } \\
\hline$R\left[F^{2}>2 \sigma\left(F^{2}\right)\right], w R\left(F^{2}\right), S$ & $0.033,0.071,0.83$ \\
\hline No. of reflections & 4156 \\
\hline No. of parameters & 289 \\
\hline $\mathrm{H}$-atom treatment & $\begin{array}{l}\mathrm{H} \text { atoms treated by a mixture of } \\
\text { independent and constrained } \\
\text { refinement }\end{array}$ \\
\hline$\Delta \rho_{\max }, \Delta \rho_{\min }\left(\mathrm{e} \AA^{-3}\right)$ & $0.22,-0.22$ \\
\hline
\end{tabular}

Computer programs: $X$-AREA (Stoe \& Cie, 2009), X-RED32 (Stoe \& Cie, 2009), SHELXS97 (Sheldrick, 2008), Mercury (Macrae et al., 2020), SHELXL2018/3 (Sheldrick, 2015), PLATON (Spek, 2020) and publCIF (Westrip, 2010).

The CIFs for compounds II-HCl (AKUXEM; Ullah \& Altaf, 2014) and VI-VIII have been updated recently in the CSD.

Compounds III to VIII all have a similar conformation; a curved or half-moon shape, as shown for the cation of $\mathbf{I} \cdot \mathbf{H C l}$ in Fig. 2. The piperidine rings of the dihydroquinoline units in compounds I.HCI, II.HCI, III, V, VII and VIII have screwboat conformations. The piperidine or piperazine rings in all eight compounds have chair conformations.

\section{Synthesis and crystallization}

The synthesis of compounds I and II has been reported (Ullah, 2012; compounds $5 c$ and $5 d$ in that paper). Colourless platelike crystals of their hydrochloride salts were obtained by slow evaporation of solutions in dichloromethane and methanol; ratios (8:3) and (8.5:1.5), respectively.

\section{Refinement}

Crystal data, data collection and structure refinement details are summarized in Table 3. The NH H atoms were located in a difference electron-density map and freely refined. The Cbound $\mathrm{H}$ atoms were included in calculated positions and refined as riding on the parent atom: $\mathrm{C}-\mathrm{H}=0.95-0.99 \AA$ with $U_{\text {iso }}(\mathrm{H})=1.2 U_{\text {eq }}(\mathrm{C})$.

\section{Acknowledgements}

HSE is grateful to the University of Neuchâtel for their support over the years.

\section{Funding information}

Funding for this research was provided by: King Fahd University of Petroleum and Minerals, Dahran, Saudia Arabia; University of Neuchâtel.

\section{References}

Feenstra, R. W., de Moes, J., Hofma, J. J., Kling, H., Kuipers, W., Long, S. K., Tulp, M. T. M., van der Heyden, J. A. M. \& Kruse, C. G. (2001). Bioorg. Med. Chem. Lett. 11, 2345-2349.

Feenstra, R. W., van den Hoogenband, A., Stroomer, C. N. J., van Stuivenberg, H. H., Tulp, M. T. M., Long, S. K., van der Heyden, J. A. M. \& Kruse, C. G. (2006). Chem. Pharm. Bull. 54, 1326-1330.

Ghani, U., Ullah, N., Ali, S. A. \& Al-Muallem, H. A. (2014). Asian J. Chem. 26, 8258-8362.

Groom, C. R., Bruno, I. J., Lightfoot, M. P. \& Ward, S. C. (2016). Acta Cryst. B72, 171-179.

Macrae, C. F., Sovago, I., Cottrell, S. J., Galek, P. T. A., McCabe, P., Pidcock, E., Platings, M., Shields, G. P., Stevens, J. S., Towler, M. \& Wood, P. A. (2020). J. Appl. Cryst. 53, 226-235.

McKinnon, J. J., Jayatilaka, D. \& Spackman, M. A. (2007). Chem. Commun. pp. 3814-3816.

Sheldrick, G. M. (2008). Acta Cryst. A64, 112-122.

Sheldrick, G. M. (2015). Acta Cryst. C71, 3-8.

Spackman, M. A. \& Jayatilaka, D. (2009). CrystEng Comm, 11, 19-32. Spek, A. L. (2020). Acta Cryst. E76, 1-11.

Stoe \& Cie. (2009). $X$-AREA and $X$-RED32. Stoe \& Cie GmbH, Darmstadt, Germany.

Tan, S. L., Jotani, M. M. \& Tiekink, E. R. T. (2019). Acta Cryst. E75, 308-318.

Turner, M. J., McKinnon, J. J., Wolff, S. K., Grimwood, D. J., Spackman, P. R., Jayatilaka, D. \& Spackman, M. A. (2017). CrystalExplorer17. University of Western Australia. http://hirshfeldsurface.net

Ullah, N. (2012). Z. Naturforsch. Teil B, 67, 75-84.

Ullah, N. (2014a). Med. Chem. 10, 484-496.

Ullah, N. (2014b). J. Enzyme Inhib. Med. Chem. 29, 281-291.

Ullah, N. \& Al-Shaheri, A. A. Q. (2012). J. Chem. Sci. 67, 253-262.

Ullah, N. \& Altaf, M. (2014). Crystallogr. Rep. 59, 1057-1062.

Ullah, N., Altaf, M. \& Mansha, M. (2017). Z. Naturforsch. Teil B, 58, 1697-1702.

Ullah, N., Altaf, M., Mansha, M. \& Ba-Salem, A. O. (2015). J. Struct. Chem. 56, 1441-1445.

Ullah, N. \& Stoeckli-Evans, H. (2014). Acta Cryst. E70, o103-o104.

Westrip, S. P. (2010). J. Appl. Cryst. 43, 920-925. 


\section{supporting information}

Acta Cryst. (2021). E77, 208-212 [https://doi.org/10.1107/S2056989021000979]

Crystal structure and Hirshfeld surface analysis of the hydrochloride salt of 8-\{4-[(6-phenylpyridin-3-yl)methyl]piperazin-1-yl\}-3,4-dihydroquinolin-2(1H)-

one

\section{Nisar Ullah and Helen Stoeckli-Evans}

Computing details

Data collection: $X$-AREA (Stoe \& Cie, 2009); cell refinement: $X$-AREA (Stoe \& Cie, 2009); data reduction: $X$-RED32

(Stoe \& Cie, 2009); program(s) used to solve structure: SHELXS97 (Sheldrick, 2008); program(s) used to refine structure: SHELXL2018/3 (Sheldrick, 2015); molecular graphics: Mercury (Macrae et al., 2020); software used to prepare material for publication: SHELXL2018/3 (Sheldrick, 2015), PLATON (Spek, 2020) and publCIF (Westrip, 2010).

4-(2-Oxo-1,2,3,4-tetrahydroquinolin-8-yl)-1-[(6-phenylpyridin-3-yl)methyl]piperazin-1-ium chloride

Crystal data

$\mathrm{C}_{25} \mathrm{H}_{27} \mathrm{~N}_{4} \mathrm{O}^{+} \cdot \mathrm{Cl}^{-}$

$M_{r}=434.95$

Triclinic, $P \overline{1}$

$a=8.4791(8) \AA$

$b=10.4091(10) \AA$

$c=13.6862(14) \AA$

$\alpha=90.138(8)^{\circ}$

$\beta=94.833(8)^{\circ}$

$\gamma=113.745(7)^{\circ}$

$V=1100.88(19) \AA^{3}$

\section{Data collection}

Stoe IPDS 2

diffractometer

Radiation source: fine-focus sealed tube

Plane graphite monochromator

$\varphi+\omega$ scans

Absorption correction: multi-scan

(MULABS; Spek, 2020)

$T_{\min }=0.379, T_{\max }=1.000$

Refinement

Refinement on $F^{2}$

Least-squares matrix: full

$R\left[F^{2}>2 \sigma\left(F^{2}\right)\right]=0.033$

$w R\left(F^{2}\right)=0.071$

$S=0.83$

4156 reflections

289 parameters
$Z=2$

$F(000)=460$

$D_{\mathrm{x}}=1.312 \mathrm{Mg} \mathrm{m}^{-3}$

Mo $K \alpha$ radiation, $\lambda=0.71073 \AA$

Cell parameters from 9997 reflections

$\theta=1.5-26.1^{\circ}$

$\mu=0.20 \mathrm{~mm}^{-1}$

$T=173 \mathrm{~K}$

Rod, colourless

$0.45 \times 0.33 \times 0.18 \mathrm{~mm}$

13410 measured reflections

4156 independent reflections

2912 reflections with $I>2 \sigma(I)$

$R_{\text {int }}=0.077$

$\theta_{\max }=25.6^{\circ}, \theta_{\min }=1.5^{\circ}$

$h=-10 \rightarrow 9$

$k=-12 \rightarrow 12$

$l=-16 \rightarrow 16$

0 restraints

Primary atom site location: structure-invariant direct methods

Secondary atom site location: difference Fourier map

Hydrogen site location: mixed 
$\mathrm{H}$ atoms treated by a mixture of independent and constrained refinement

$w=1 /\left[\sigma^{2}\left(F_{\mathrm{o}}^{2}\right)+(0.0262 P)^{2}\right]$

where $P=\left(F_{\mathrm{o}}^{2}+2 F_{\mathrm{c}}^{2}\right) / 3$

$(\Delta / \sigma)_{\max }=0.001$

$\Delta \rho_{\max }=0.22$ e $\AA^{-3}$
$\Delta \rho_{\min }=-0.22$ e $\AA^{-3}$

Extinction correction: (SHELXL-2018/3;

Sheldrick, 2015),

$\mathrm{Fc}^{*}=\mathrm{kFc}\left[1+0.001 \times \mathrm{xc}^{2} \lambda^{3} / \sin (2 \theta)\right]^{-1 / 4}$

Extinction coefficient: 0.0061 (11)

\section{Special details}

Geometry. All esds (except the esd in the dihedral angle between two 1.s. planes) are estimated using the full covariance matrix. The cell esds are taken into account individually in the estimation of esds in distances, angles and torsion angles; correlations between esds in cell parameters are only used when they are defined by crystal symmetry. An approximate (isotropic) treatment of cell esds is used for estimating esds involving 1.s. planes.

Fractional atomic coordinates and isotropic or equivalent isotropic displacement parameters $\left(\AA^{2}\right)$

\begin{tabular}{|c|c|c|c|c|}
\hline & $x$ & $y$ & $z$ & $U_{\text {iso }} * / U_{\text {eq }}$ \\
\hline $\mathrm{O} 1$ & $-0.17052(13)$ & $0.47203(11)$ & $-0.07307(8)$ & 0.0288 \\
\hline N1 & $0.07882(16)$ & $0.49233(13)$ & $-0.13301(10)$ & $0.0234(3)$ \\
\hline $\mathrm{H} 1 \mathrm{~N}$ & $0.122(2)$ & $0.5073(18)$ & $-0.0734(13)$ & $0.034(5)^{*}$ \\
\hline $\mathrm{N} 2$ & 0.44879 (14) & $0.63611(12)$ & $-0.11540(9)$ & $0.0220(3)$ \\
\hline N3 & $0.68062(15)$ & $0.82122(13)$ & $0.04180(9)$ & $0.0217(3)$ \\
\hline $\mathrm{H} 3 \mathrm{~N}$ & $0.746(2)$ & $0.906(2)$ & $0.0090(13)$ & $0.052(5)^{*}$ \\
\hline N4 & $0.94060(18)$ & $1.20937(14)$ & $0.25205(11)$ & $0.0399(4)$ \\
\hline $\mathrm{C} 1$ & $-0.09235(18)$ & $0.46487(15)$ & $-0.14318(11)$ & $0.0236(3)$ \\
\hline $\mathrm{C} 2$ & $-0.1799(2)$ & $0.42765(18)$ & $-0.24529(12)$ & $0.0329(4)$ \\
\hline $\mathrm{H} 2 \mathrm{~A}$ & -0.160759 & 0.514512 & -0.280987 & $0.040 *$ \\
\hline $\mathrm{H} 2 \mathrm{~B}$ & -0.306002 & 0.375119 & -0.242625 & $0.040 *$ \\
\hline $\mathrm{C} 3$ & $-0.1077(2)$ & 0.33797 (19) & $-0.29954(13)$ & $0.0392(4)$ \\
\hline $\mathrm{H} 3 \mathrm{~A}$ & -0.135909 & 0.247286 & -0.267359 & $0.047 *$ \\
\hline H3B & -0.160866 & 0.318222 & -0.368136 & $0.047^{*}$ \\
\hline $\mathrm{C} 4$ & $0.0858(2)$ & $0.41520(16)$ & $-0.29844(12)$ & $0.0302(4)$ \\
\hline $\mathrm{C} 5$ & $0.1751(2)$ & 0.41199 (19) & $-0.37763(13)$ & $0.0392(4)$ \\
\hline H5 & 0.114179 & 0.360241 & -0.436140 & $0.047 *$ \\
\hline C6 & $0.3535(2)$ & $0.48412(19)$ & $-0.37196(13)$ & $0.0392(4)$ \\
\hline H6 & 0.414790 & 0.480812 & -0.426264 & $0.047 *$ \\
\hline $\mathrm{C} 7$ & $0.4424(2)$ & $0.56092(17)$ & $-0.28716(12)$ & $0.0310(4)$ \\
\hline $\mathrm{H} 7$ & 0.564389 & 0.610806 & -0.284514 & $0.037^{*}$ \\
\hline $\mathrm{C} 8$ & $0.35719(18)$ & $0.56693(15)$ & $-0.20549(11)$ & 0.0230 \\
\hline $\mathrm{C} 9$ & $0.17520(18)$ & $0.49231(15)$ & $-0.21240(11)$ & 0.0228 \\
\hline $\mathrm{C} 10$ & $0.63546(18)$ & $0.67238(16)$ & $-0.10867(11)$ & $0.0266(4)$ \\
\hline $\mathrm{H} 10 \mathrm{~A}$ & 0.692624 & 0.755875 & -0.147562 & $0.032 *$ \\
\hline H10B & 0.656720 & 0.593449 & -0.136332 & $0.032 *$ \\
\hline $\mathrm{C} 11$ & $0.71077(19)$ & $0.70267(16)$ & $-0.00407(11)$ & $0.0256(4)$ \\
\hline H11A & 0.836721 & 0.727272 & -0.001024 & $0.031 *$ \\
\hline H11B & 0.657845 & 0.617257 & 0.033766 & $0.031 *$ \\
\hline $\mathrm{C} 12$ & $0.49249(17)$ & $0.79433(16)$ & $0.02658(11)$ & 0.0238 \\
\hline $\mathrm{H} 12 \mathrm{~A}$ & 0.476973 & 0.878517 & 0.049257 & $0.029 *$ \\
\hline H12B & 0.425884 & 0.715170 & 0.066760 & $0.029 *$ \\
\hline C13 & $0.42172(18)$ & $0.75986(15)$ & $-0.08004(11)$ & 0.0232 \\
\hline
\end{tabular}




$\begin{array}{lllll}\text { H13A } & 0.296647 & 0.739395 & -0.086798 & 0.028^{*} \\ \text { H13B } & 0.481455 & 0.841377 & -0.120110 & 0.028^{*} \\ \text { C14 } & 0.74694(19) & 0.83801(16) & 0.14807(11) & 0.0271(4) \\ \text { H14A } & 0.863559 & 0.836905 & 0.153532 & 0.033^{*} \\ \text { H14B } & 0.670376 & 0.755999 & 0.182549 & 0.033^{*} \\ \text { C15 } & 0.75825(19) & 0.96984(16) & 0.19971(11) & 0.0259(4) \\ \text { C16 } & 0.6286(2) & 0.97397(16) & 0.25369(11) & 0.0276(4) \\ \text { H16 } & 0.521948 & 0.894116 & 0.253316 & 0.033^{*} \\ \text { C17 } & 0.6549(2) & 1.09519(16) & 0.30836(11) & 0.0289(4) \\ \text { C18 } & 0.8145(2) & 1.20792(17) & 0.30499(12) & 0.0354(4) \\ \text { H18 } & 0.835305 & 1.290278 & 0.343377 & 0.042^{*} \\ \text { C19 } & 0.9109(2) & 1.09080(17) & 0.20072(13) & 0.0351(4) \\ \text { H19 } & 0.998875 & 1.089041 & 0.162807 & 0.042^{*} \\ \text { C20 } & 0.5209(2) & 1.10509(17) & 0.36734(12) & 0.0326(4) \\ \text { C21 } & 0.3467(2) & 1.04802(18) & 0.33182(13) & 0.0366(4) \\ \text { H21 } & 0.312714 & 1.001744 & 0.268836 & 0.044^{*} \\ \text { C22 } & 0.2225(3) & 1.0581(2) & 0.38747(14) & 0.0480(5) \\ \text { H22 } & 0.103978 & 1.017354 & 0.362924 & 0.058^{*} \\ \text { C23 } & 0.2710(3) & 1.1276(2) & 0.47900(15) & 0.0557(6) \\ \text { H23 } & 0.186196 & 1.135071 & 0.517087 & 0.067^{*} \\ \text { C24 } & 0.4438(3) & 1.1856(2) & 0.51400(14) & 0.0515(5) \\ \text { H24 } & 0.477753 & 1.233593 & 0.576400 & 0.062^{*} \\ \text { C25 } & 0.5676(3) & 1.17463(19) & 0.45928(13) & 0.0436(5) \\ \text { H25 } & 0.685829 & 1.214852 & 0.484499 & 0.052^{*} \\ \text { C11 } & 0.16918(5) & 0.91117(4) & 0.07387(3) & 0.02965(12)\end{array}$

Atomic displacement parameters $\left(\AA^{2}\right)$

\begin{tabular}{lllllll}
\hline & $U^{11}$ & $U^{22}$ & $U^{33}$ & $U^{12}$ & $U^{13}$ & $U^{23}$ \\
\hline $\mathrm{O} 1$ & $0.0214(5)$ & $0.0315(6)$ & $0.0325(6)$ & $0.0094(5)$ & $0.0034(5)$ & $0.0009(5)$ \\
$\mathrm{N} 1$ & $0.0193(6)$ & $0.0251(7)$ & $0.0233(8)$ & $0.0067(5)$ & $0.0005(6)$ & $-0.0016(6)$ \\
$\mathrm{N} 2$ & $0.0166(6)$ & $0.0211(7)$ & $0.0291(7)$ & $0.0082(5)$ & $0.0028(5)$ & $-0.0021(5)$ \\
$\mathrm{N} 3$ & $0.0168(6)$ & $0.0190(6)$ & $0.0284(7)$ & $0.0063(5)$ & $0.0026(5)$ & $0.0015(5)$ \\
$\mathrm{N} 4$ & $0.0338(8)$ & $0.0276(8)$ & $0.0486(9)$ & $0.0042(6)$ & $-0.0047(7)$ & $-0.0027(7)$ \\
$\mathrm{C} 1$ & $0.0190(7)$ & $0.0168(7)$ & $0.0326(9)$ & $0.0045(6)$ & $0.0034(7)$ & $0.0017(6)$ \\
$\mathrm{C} 2$ & $0.0223(8)$ & $0.0363(10)$ & $0.0332(9)$ & $0.0056(7)$ & $-0.0024(7)$ & $-0.0034(8)$ \\
$\mathrm{C} 3$ & $0.0324(9)$ & $0.0379(10)$ & $0.0365(10)$ & $0.0046(8)$ & $-0.0038(8)$ & $-0.0151(8)$ \\
$\mathrm{C} 4$ & $0.0314(9)$ & $0.0255(9)$ & $0.0318(9)$ & $0.0100(7)$ & $0.0002(7)$ & $-0.0055(7)$ \\
$\mathrm{C} 5$ & $0.0440(10)$ & $0.0421(11)$ & $0.0304(10)$ & $0.0168(8)$ & $0.0004(8)$ & $-0.0131(8)$ \\
C6 & $0.0448(11)$ & $0.0452(11)$ & $0.0306(10)$ & $0.0196(9)$ & $0.0129(8)$ & $-0.0062(8)$ \\
C7 & $0.0277(8)$ & $0.0314(9)$ & $0.0346(9)$ & $0.0120(7)$ & $0.0074(7)$ & $-0.0004(7)$ \\
C8 & $0.0253(8)$ & $0.0197(8)$ & $0.0259(8)$ & $0.0109(6)$ & $0.0035(7)$ & $0.0009(6)$ \\
C9 & $0.0253(8)$ & $0.0182(7)$ & $0.0255(8)$ & $0.0089(6)$ & $0.0037(7)$ & $-0.0004(6)$ \\
C10 & $0.0182(7)$ & $0.0260(8)$ & $0.0366(9)$ & $0.0093(6)$ & $0.0059(7)$ & $-0.0005(7)$ \\
C11 & $0.0184(7)$ & $0.0223(8)$ & $0.0383(10)$ & $0.0105(6)$ & $0.0021(7)$ & $-0.0009(7)$ \\
C12 & $0.0152(7)$ & $0.0248(8)$ & $0.0313(9)$ & $0.0079(6)$ & $0.0029(6)$ & $-0.0023(6)$ \\
C13 & $0.0183(7)$ & $0.0217(8)$ & $0.0302(9)$ & $0.0091(6)$ & $0.0007(6)$ & $-0.0018(6)$ \\
C14 & $0.0217(8)$ & $0.0273(9)$ & $0.0311(9)$ & $0.0092(6)$ & $-0.0006(7)$ & $0.0032(7)$
\end{tabular}




\begin{tabular}{lllllll} 
C15 & $0.0261(8)$ & $0.0246(8)$ & $0.0242(8)$ & $0.0085(6)$ & $-0.0037(7)$ & $0.0011(6)$ \\
C16 & $0.0281(8)$ & $0.0232(8)$ & $0.0267(9)$ & $0.0063(6)$ & $-0.0019(7)$ & $0.0001(7)$ \\
C17 & $0.0379(9)$ & $0.0271(9)$ & $0.0216(9)$ & $0.0144(7)$ & $-0.0041(7)$ & $-0.0001(7)$ \\
C18 & $0.0448(10)$ & $0.0227(9)$ & $0.0325(10)$ & $0.0099(7)$ & $-0.0103(8)$ & $-0.0043(7)$ \\
C19 & $0.0278(9)$ & $0.0305(10)$ & $0.0404(10)$ & $0.0057(7)$ & $-0.0010(8)$ & $-0.0003(8)$ \\
C20 & $0.0492(11)$ & $0.0253(9)$ & $0.0252(9)$ & $0.0179(8)$ & $-0.0002(8)$ & $0.0013(7)$ \\
C21 & $0.0497(11)$ & $0.0336(10)$ & $0.0296(9)$ & $0.0197(8)$ & $0.0049(8)$ & $0.0003(7)$ \\
C22 & $0.0534(12)$ & $0.0526(12)$ & $0.0471(12)$ & $0.0299(10)$ & $0.0099(10)$ & $0.0062(10)$ \\
C23 & $0.0860(17)$ & $0.0597(14)$ & $0.0428(13)$ & $0.0481(13)$ & $0.0242(12)$ & $0.0077(10)$ \\
C24 & $0.0872(17)$ & $0.0492(12)$ & $0.0286(11)$ & $0.0380(12)$ & $0.0067(11)$ & $-0.0037(9)$ \\
C25 & $0.0672(13)$ & $0.0374(10)$ & $0.0294(10)$ & $0.0259(9)$ & $-0.0023(9)$ & $-0.0025(8)$ \\
C11 & $0.02283(19)$ & $0.0261(2)$ & $0.0381(2)$ & $0.00758(15)$ & $0.00489(17)$ & $0.00481(17)$ \\
\hline
\end{tabular}

Geometric parameters $\left(\AA,{ }^{\circ}\right)$

\begin{tabular}{|c|c|c|c|}
\hline $\mathrm{O} 1-\mathrm{C} 1$ & $1.2298(17)$ & $\mathrm{C} 10-\mathrm{H} 10 \mathrm{~B}$ & 0.9900 \\
\hline $\mathrm{N} 1-\mathrm{C} 1$ & $1.3562(19)$ & $\mathrm{C} 11-\mathrm{H} 11 \mathrm{~A}$ & 0.9900 \\
\hline $\mathrm{N} 1-\mathrm{C} 9$ & $1.4136(19)$ & $\mathrm{C} 11-\mathrm{H} 11 \mathrm{~B}$ & 0.9900 \\
\hline $\mathrm{N} 1-\mathrm{H} 1 \mathrm{~N}$ & $0.852(17)$ & $\mathrm{C} 12-\mathrm{C} 13$ & $1.515(2)$ \\
\hline N2-C 8 & $1.4233(19)$ & $\mathrm{C} 12-\mathrm{H} 12 \mathrm{~A}$ & 0.9900 \\
\hline $\mathrm{N} 2-\mathrm{C} 10$ & $1.4665(18)$ & $\mathrm{C} 12-\mathrm{H} 12 \mathrm{~B}$ & 0.9900 \\
\hline $\mathrm{N} 2-\mathrm{C} 13$ & $1.4832(19)$ & $\mathrm{C} 13-\mathrm{H} 13 \mathrm{~A}$ & 0.9900 \\
\hline N3-C14 & $1.4981(19)$ & $\mathrm{C} 13-\mathrm{H} 13 \mathrm{~B}$ & 0.9900 \\
\hline $\mathrm{N} 3-\mathrm{C} 12$ & $1.5002(17)$ & $\mathrm{C} 14-\mathrm{C} 15$ & $1.507(2)$ \\
\hline $\mathrm{N} 3-\mathrm{C} 11$ & $1.5031(19)$ & $\mathrm{C} 14-\mathrm{H} 14 \mathrm{~A}$ & 0.9900 \\
\hline $\mathrm{N} 3-\mathrm{H} 3 \mathrm{~N}$ & $0.967(18)$ & $\mathrm{C} 14-\mathrm{H} 14 \mathrm{~B}$ & 0.9900 \\
\hline $\mathrm{N} 4-\mathrm{C} 18$ & $1.336(2)$ & $\mathrm{C} 15-\mathrm{C} 16$ & $1.390(2)$ \\
\hline N4 - C19 & $1.340(2)$ & $\mathrm{C} 15-\mathrm{C} 19$ & $1.395(2)$ \\
\hline $\mathrm{C} 1-\mathrm{C} 2$ & $1.498(2)$ & $\mathrm{C} 16-\mathrm{C} 17$ & $1.394(2)$ \\
\hline $\mathrm{C} 2-\mathrm{C} 3$ & $1.527(2)$ & $\mathrm{C} 16-\mathrm{H} 16$ & 0.9500 \\
\hline $\mathrm{C} 2-\mathrm{H} 2 \mathrm{~A}$ & 0.9900 & $\mathrm{C} 17-\mathrm{C} 18$ & $1.394(2)$ \\
\hline $\mathrm{C} 2-\mathrm{H} 2 \mathrm{~B}$ & 0.9900 & $\mathrm{C} 17-\mathrm{C} 20$ & $1.484(2)$ \\
\hline $\mathrm{C} 3-\mathrm{C} 4$ & $1.507(2)$ & C18-H18 & 0.9500 \\
\hline $\mathrm{C} 3-\mathrm{H} 3 \mathrm{~A}$ & 0.9900 & C19-H19 & 0.9500 \\
\hline $\mathrm{C} 3-\mathrm{H} 3 \mathrm{~B}$ & 0.9900 & $\mathrm{C} 20-\mathrm{C} 25$ & $1.395(2)$ \\
\hline $\mathrm{C} 4-\mathrm{C} 5$ & $1.381(2)$ & $\mathrm{C} 20-\mathrm{C} 21$ & $1.393(2)$ \\
\hline $\mathrm{C} 4-\mathrm{C} 9$ & $1.400(2)$ & $\mathrm{C} 21-\mathrm{C} 22$ & $1.387(2)$ \\
\hline $\mathrm{C} 5-\mathrm{C} 6$ & $1.387(2)$ & $\mathrm{C} 21-\mathrm{H} 21$ & 0.9500 \\
\hline $\mathrm{C} 5-\mathrm{H} 5$ & 0.9500 & $\mathrm{C} 22-\mathrm{C} 23$ & $1.389(3)$ \\
\hline $\mathrm{C} 6-\mathrm{C} 7$ & $1.384(2)$ & $\mathrm{C} 22-\mathrm{H} 22$ & 0.9500 \\
\hline C6-H6 & 0.9500 & $\mathrm{C} 23-\mathrm{C} 24$ & $1.382(3)$ \\
\hline $\mathrm{C} 7-\mathrm{C} 8$ & $1.395(2)$ & $\mathrm{C} 23-\mathrm{H} 23$ & 0.9500 \\
\hline $\mathrm{C} 7-\mathrm{H} 7$ & 0.9500 & $\mathrm{C} 24-\mathrm{C} 25$ & $1.380(3)$ \\
\hline $\mathrm{C} 8-\mathrm{C} 9$ & $1.415(2)$ & $\mathrm{C} 24-\mathrm{H} 24$ & 0.9500 \\
\hline $\mathrm{C} 10-\mathrm{C} 11$ & $1.496(2)$ & $\mathrm{C} 25-\mathrm{H} 25$ & 0.9500 \\
\hline $\mathrm{C} 10-\mathrm{H} 10 \mathrm{~A}$ & 0.9900 & & \\
\hline $\mathrm{C} 1-\mathrm{N} 1-\mathrm{C} 9$ & $123.74(14)$ & $\mathrm{C} 10-\mathrm{C} 11-\mathrm{H} 11 \mathrm{~B}$ & 109.3 \\
\hline
\end{tabular}




\begin{tabular}{|c|c|c|c|}
\hline $\mathrm{C} 1-\mathrm{N} 1-\mathrm{H} 1 \mathrm{~N}$ & $112.8(11)$ & $\mathrm{N} 3-\mathrm{C} 11-\mathrm{H} 11 \mathrm{~B}$ & 109.3 \\
\hline $\mathrm{C} 9-\mathrm{N} 1-\mathrm{H} 1 \mathrm{~N}$ & $123.5(11)$ & $\mathrm{H} 11 \mathrm{~A}-\mathrm{C} 11-\mathrm{H} 11 \mathrm{~B}$ & 107.9 \\
\hline $\mathrm{C} 8-\mathrm{N} 2-\mathrm{C} 10$ & $114.51(12)$ & $\mathrm{N} 3-\mathrm{C} 12-\mathrm{C} 13$ & $112.12(12)$ \\
\hline $\mathrm{C} 8-\mathrm{N} 2-\mathrm{C} 13$ & $118.19(12)$ & $\mathrm{N} 3-\mathrm{C} 12-\mathrm{H} 12 \mathrm{~A}$ & 109.2 \\
\hline $\mathrm{C} 10-\mathrm{N} 2-\mathrm{C} 13$ & $108.27(11)$ & $\mathrm{C} 13-\mathrm{C} 12-\mathrm{H} 12 \mathrm{~A}$ & 109.2 \\
\hline $\mathrm{C} 14-\mathrm{N} 3-\mathrm{C} 12$ & $112.69(12)$ & $\mathrm{N} 3-\mathrm{C} 12-\mathrm{H} 12 \mathrm{~B}$ & 109.2 \\
\hline $\mathrm{C} 14-\mathrm{N} 3-\mathrm{C} 11$ & $108.66(12)$ & $\mathrm{C} 13-\mathrm{C} 12-\mathrm{H} 12 \mathrm{~B}$ & 109.2 \\
\hline $\mathrm{C} 12-\mathrm{N} 3-\mathrm{C} 11$ & $110.14(11)$ & $\mathrm{H} 12 \mathrm{~A}-\mathrm{C} 12-\mathrm{H} 12 \mathrm{~B}$ & 107.9 \\
\hline $\mathrm{C} 14-\mathrm{N} 3-\mathrm{H} 3 \mathrm{~N}$ & $109.2(11)$ & $\mathrm{N} 2-\mathrm{C} 13-\mathrm{C} 12$ & $109.53(12)$ \\
\hline $\mathrm{C} 12-\mathrm{N} 3-\mathrm{H} 3 \mathrm{~N}$ & $108.5(11)$ & $\mathrm{N} 2-\mathrm{C} 13-\mathrm{H} 13 \mathrm{~A}$ & 109.8 \\
\hline $\mathrm{C} 11-\mathrm{N} 3-\mathrm{H} 3 \mathrm{~N}$ & $107.6(11)$ & $\mathrm{C} 12-\mathrm{C} 13-\mathrm{H} 13 \mathrm{~A}$ & 109.8 \\
\hline $\mathrm{C} 18-\mathrm{N} 4-\mathrm{C} 19$ & $116.51(14)$ & $\mathrm{N} 2-\mathrm{C} 13-\mathrm{H} 13 \mathrm{~B}$ & 109.8 \\
\hline $\mathrm{O} 1-\mathrm{C} 1-\mathrm{N} 1$ & $122.03(14)$ & $\mathrm{C} 12-\mathrm{C} 13-\mathrm{H} 13 \mathrm{~B}$ & 109.8 \\
\hline $\mathrm{O} 1-\mathrm{C} 1-\mathrm{C} 2$ & $121.99(13)$ & $\mathrm{H} 13 \mathrm{~A}-\mathrm{C} 13-\mathrm{H} 13 \mathrm{~B}$ & 108.2 \\
\hline $\mathrm{N} 1-\mathrm{C} 1-\mathrm{C} 2$ & $115.97(14)$ & N3-C14-C15 & $115.00(13)$ \\
\hline $\mathrm{C} 1-\mathrm{C} 2-\mathrm{C} 3$ & $110.04(14)$ & N3-C14-H14A & 108.5 \\
\hline $\mathrm{C} 1-\mathrm{C} 2-\mathrm{H} 2 \mathrm{~A}$ & 109.7 & $\mathrm{C} 15-\mathrm{C} 14-\mathrm{H} 14 \mathrm{~A}$ & 108.5 \\
\hline $\mathrm{C} 3-\mathrm{C} 2-\mathrm{H} 2 \mathrm{~A}$ & 109.7 & $\mathrm{~N} 3-\mathrm{C} 14-\mathrm{H} 14 \mathrm{~B}$ & 108.5 \\
\hline $\mathrm{C} 1-\mathrm{C} 2-\mathrm{H} 2 \mathrm{~B}$ & 109.7 & $\mathrm{C} 15-\mathrm{C} 14-\mathrm{H} 14 \mathrm{~B}$ & 108.5 \\
\hline $\mathrm{C} 3-\mathrm{C} 2-\mathrm{H} 2 \mathrm{~B}$ & 109.7 & $\mathrm{H} 14 \mathrm{~A}-\mathrm{C} 14-\mathrm{H} 14 \mathrm{~B}$ & 107.5 \\
\hline $\mathrm{H} 2 \mathrm{~A}-\mathrm{C} 2-\mathrm{H} 2 \mathrm{~B}$ & 108.2 & $\mathrm{C} 16-\mathrm{C} 15-\mathrm{C} 19$ & $117.83(15)$ \\
\hline $\mathrm{C} 4-\mathrm{C} 3-\mathrm{C} 2$ & $109.40(13)$ & $\mathrm{C} 16-\mathrm{C} 15-\mathrm{C} 14$ & $122.94(13)$ \\
\hline $\mathrm{C} 4-\mathrm{C} 3-\mathrm{H} 3 \mathrm{~A}$ & 109.8 & $\mathrm{C} 19-\mathrm{C} 15-\mathrm{C} 14$ & $119.00(15)$ \\
\hline $\mathrm{C} 2-\mathrm{C} 3-\mathrm{H} 3 \mathrm{~A}$ & 109.8 & $\mathrm{C} 15-\mathrm{C} 16-\mathrm{C} 17$ & $120.04(14)$ \\
\hline $\mathrm{C} 4-\mathrm{C} 3-\mathrm{H} 3 \mathrm{~B}$ & 109.8 & $\mathrm{C} 15-\mathrm{C} 16-\mathrm{H} 16$ & 120.0 \\
\hline $\mathrm{C} 2-\mathrm{C} 3-\mathrm{H} 3 \mathrm{~B}$ & 109.8 & $\mathrm{C} 17-\mathrm{C} 16-\mathrm{H} 16$ & 120.0 \\
\hline $\mathrm{H} 3 \mathrm{~A}-\mathrm{C} 3-\mathrm{H} 3 \mathrm{~B}$ & 108.2 & $\mathrm{C} 18-\mathrm{C} 17-\mathrm{C} 16$ & $116.57(15)$ \\
\hline $\mathrm{C} 5-\mathrm{C} 4-\mathrm{C} 9$ & $119.99(15)$ & $\mathrm{C} 18-\mathrm{C} 17-\mathrm{C} 20$ & $121.22(15)$ \\
\hline $\mathrm{C} 5-\mathrm{C} 4-\mathrm{C} 3$ & $122.79(15)$ & $\mathrm{C} 16-\mathrm{C} 17-\mathrm{C} 20$ & $122.21(14)$ \\
\hline $\mathrm{C} 9-\mathrm{C} 4-\mathrm{C} 3$ & $117.22(14)$ & $\mathrm{N} 4-\mathrm{C} 18-\mathrm{C} 17$ & $125.19(16)$ \\
\hline $\mathrm{C} 4-\mathrm{C} 5-\mathrm{C} 6$ & $120.19(16)$ & N4-C18-H18 & 117.4 \\
\hline $\mathrm{C} 4-\mathrm{C} 5-\mathrm{H} 5$ & 119.9 & $\mathrm{C} 17-\mathrm{C} 18-\mathrm{H} 18$ & 117.4 \\
\hline $\mathrm{C} 6-\mathrm{C} 5-\mathrm{H} 5$ & 119.9 & $\mathrm{~N} 4-\mathrm{C} 19-\mathrm{C} 15$ & $123.81(17)$ \\
\hline $\mathrm{C} 7-\mathrm{C} 6-\mathrm{C} 5$ & $119.99(15)$ & $\mathrm{N} 4-\mathrm{C} 19-\mathrm{H} 19$ & 118.1 \\
\hline $\mathrm{C} 7-\mathrm{C} 6-\mathrm{H} 6$ & 120.0 & $\mathrm{C} 15-\mathrm{C} 19-\mathrm{H} 19$ & 118.1 \\
\hline $\mathrm{C} 5-\mathrm{C} 6-\mathrm{H} 6$ & 120.0 & $\mathrm{C} 25-\mathrm{C} 20-\mathrm{C} 21$ & $118.47(17)$ \\
\hline $\mathrm{C} 6-\mathrm{C} 7-\mathrm{C} 8$ & $121.68(14)$ & $\mathrm{C} 25-\mathrm{C} 20-\mathrm{C} 17$ & $120.38(17)$ \\
\hline $\mathrm{C} 6-\mathrm{C} 7-\mathrm{H} 7$ & 119.2 & $\mathrm{C} 21-\mathrm{C} 20-\mathrm{C} 17$ & $121.13(15)$ \\
\hline $\mathrm{C} 8-\mathrm{C} 7-\mathrm{H} 7$ & 119.2 & $\mathrm{C} 22-\mathrm{C} 21-\mathrm{C} 20$ & $120.67(18)$ \\
\hline $\mathrm{C} 7-\mathrm{C} 8-\mathrm{N} 2$ & $121.99(13)$ & $\mathrm{C} 22-\mathrm{C} 21-\mathrm{H} 21$ & 119.7 \\
\hline $\mathrm{C} 7-\mathrm{C} 8-\mathrm{C} 9$ & $117.59(14)$ & $\mathrm{C} 20-\mathrm{C} 21-\mathrm{H} 21$ & 119.7 \\
\hline $\mathrm{N} 2-\mathrm{C} 8-\mathrm{C} 9$ & $120.26(13)$ & $\mathrm{C} 21-\mathrm{C} 22-\mathrm{C} 23$ & $120.2(2)$ \\
\hline $\mathrm{C} 4-\mathrm{C} 9-\mathrm{N} 1$ & $118.14(13)$ & $\mathrm{C} 21-\mathrm{C} 22-\mathrm{H} 22$ & 119.9 \\
\hline $\mathrm{C} 4-\mathrm{C} 9-\mathrm{C} 8$ & $120.56(14)$ & $\mathrm{C} 23-\mathrm{C} 22-\mathrm{H} 22$ & 119.9 \\
\hline $\mathrm{N} 1-\mathrm{C} 9-\mathrm{C} 8$ & $121.30(13)$ & $\mathrm{C} 24-\mathrm{C} 23-\mathrm{C} 22$ & $119.25(19)$ \\
\hline $\mathrm{N} 2-\mathrm{C} 10-\mathrm{C} 11$ & $110.45(12)$ & $\mathrm{C} 24-\mathrm{C} 23-\mathrm{H} 23$ & 120.4 \\
\hline $\mathrm{N} 2-\mathrm{C} 10-\mathrm{H} 10 \mathrm{~A}$ & 109.6 & $\mathrm{C} 22-\mathrm{C} 23-\mathrm{H} 23$ & 120.4 \\
\hline $\mathrm{C} 11-\mathrm{C} 10-\mathrm{H} 10 \mathrm{~A}$ & 109.6 & $\mathrm{C} 25-\mathrm{C} 24-\mathrm{C} 23$ & $120.72(19)$ \\
\hline
\end{tabular}




\begin{tabular}{|c|c|}
\hline $\mathrm{N} 2-\mathrm{C} 10-\mathrm{H} 10 \mathrm{~B}$ & 109.6 \\
\hline $\mathrm{C} 11-\mathrm{C} 10-\mathrm{H} 10 \mathrm{~B}$ & 109.6 \\
\hline $\mathrm{H} 10 \mathrm{~A}-\mathrm{C} 10-\mathrm{H} 10 \mathrm{~B}$ & 108.1 \\
\hline $\mathrm{C} 10-\mathrm{C} 11-\mathrm{N} 3$ & $111.72(13)$ \\
\hline $\mathrm{C} 10-\mathrm{C} 11-\mathrm{H} 11 \mathrm{~A}$ & 109.3 \\
\hline $\mathrm{N} 3-\mathrm{C} 11-\mathrm{H} 11 \mathrm{~A}$ & 109.3 \\
\hline $\mathrm{C} 9-\mathrm{N} 1-\mathrm{C} 1-\mathrm{O} 1$ & $-176.62(13)$ \\
\hline $\mathrm{C} 9-\mathrm{N} 1-\mathrm{C} 1-\mathrm{C} 2$ & $2.4(2)$ \\
\hline $\mathrm{O} 1-\mathrm{C} 1-\mathrm{C} 2-\mathrm{C} 3$ & $-143.54(15)$ \\
\hline $\mathrm{N} 1-\mathrm{C} 1-\mathrm{C} 2-\mathrm{C} 3$ & $37.46(18)$ \\
\hline $\mathrm{C} 1-\mathrm{C} 2-\mathrm{C} 3-\mathrm{C} 4$ & $-56.18(18)$ \\
\hline $\mathrm{C} 2-\mathrm{C} 3-\mathrm{C} 4-\mathrm{C} 5$ & $-141.79(17)$ \\
\hline $\mathrm{C} 2-\mathrm{C} 3-\mathrm{C} 4-\mathrm{C} 9$ & $38.3(2)$ \\
\hline $\mathrm{C} 9-\mathrm{C} 4-\mathrm{C} 5-\mathrm{C} 6$ & $0.5(3)$ \\
\hline $\mathrm{C} 3-\mathrm{C} 4-\mathrm{C} 5-\mathrm{C} 6$ & $-179.36(17)$ \\
\hline $\mathrm{C} 4-\mathrm{C} 5-\mathrm{C} 6-\mathrm{C} 7$ & $-0.7(3)$ \\
\hline $\mathrm{C} 5-\mathrm{C} 6-\mathrm{C} 7-\mathrm{C} 8$ & $0.8(3)$ \\
\hline $\mathrm{C} 6-\mathrm{C} 7-\mathrm{C} 8-\mathrm{N} 2$ & $174.79(15)$ \\
\hline $\mathrm{C} 6-\mathrm{C} 7-\mathrm{C} 8-\mathrm{C} 9$ & $-0.5(2)$ \\
\hline $\mathrm{C} 10-\mathrm{N} 2-\mathrm{C} 8-\mathrm{C} 7$ & $-12.7(2)$ \\
\hline $\mathrm{C} 13-\mathrm{N} 2-\mathrm{C} 8-\mathrm{C} 7$ & $116.78(16)$ \\
\hline $\mathrm{C} 10-\mathrm{N} 2-\mathrm{C} 8-\mathrm{C} 9$ & $162.54(13)$ \\
\hline $\mathrm{C} 13-\mathrm{N} 2-\mathrm{C} 8-\mathrm{C} 9$ & $-68.01(17)$ \\
\hline $\mathrm{C} 5-\mathrm{C} 4-\mathrm{C} 9-\mathrm{N} 1$ & $-179.77(15)$ \\
\hline $\mathrm{C} 3-\mathrm{C} 4-\mathrm{C} 9-\mathrm{N} 1$ & $0.1(2)$ \\
\hline $\mathrm{C} 5-\mathrm{C} 4-\mathrm{C} 9-\mathrm{C} 8$ & $-0.3(2)$ \\
\hline $\mathrm{C} 3-\mathrm{C} 4-\mathrm{C} 9-\mathrm{C} 8$ & $179.60(15)$ \\
\hline $\mathrm{C} 1-\mathrm{N} 1-\mathrm{C} 9-\mathrm{C} 4$ & $-23.1(2)$ \\
\hline $\mathrm{C} 1-\mathrm{N} 1-\mathrm{C} 9-\mathrm{C} 8$ & $157.43(14)$ \\
\hline $\mathrm{C} 7-\mathrm{C} 8-\mathrm{C} 9-\mathrm{C} 4$ & $0.3(2)$ \\
\hline $\mathrm{N} 2-\mathrm{C} 8-\mathrm{C} 9-\mathrm{C} 4$ & $-175.13(14)$ \\
\hline $\mathrm{C} 7-\mathrm{C} 8-\mathrm{C} 9-\mathrm{N} 1$ & $179.77(14)$ \\
\hline $\mathrm{N} 2-\mathrm{C} 8-\mathrm{C} 9-\mathrm{N} 1$ & $4.4(2)$ \\
\hline $\mathrm{C} 8-\mathrm{N} 2-\mathrm{C} 10-\mathrm{C} 11$ & $-162.59(12)$ \\
\hline $\mathrm{C} 13-\mathrm{N} 2-\mathrm{C} 10-\mathrm{C} 11$ & $63.20(15)$ \\
\hline $\mathrm{N} 2-\mathrm{C} 10-\mathrm{C} 11-\mathrm{N} 3$ & $-58.73(16)$ \\
\hline $\mathrm{C} 14-\mathrm{N} 3-\mathrm{C} 11-\mathrm{C} 10$ & $175.64(11)$ \\
\hline $\mathrm{C} 12-\mathrm{N} 3-\mathrm{C} 11-\mathrm{C} 10$ & $51.76(15)$ \\
\hline
\end{tabular}

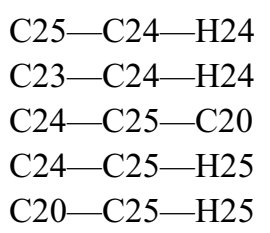

119.6

119.6

120.67 (19)

119.7

119.7

$-172.97(12)$

$-51.46(16)$

$165.68(11)$

$-62.04(14)$

$57.34(15)$

$-69.10(16)$

$168.56(12)$

$95.52(17)$

$-90.00(17)$

$-1.5(2)$

$173.04(14)$

0.1 (2)

$-179.93(14)$

$-1.9(3)$

1.7 (2)

$-178.24(15)$

$0.4(3)$

$1.3(3)$

$-173.47(16)$

$-39.3(2)$

$140.79(17)$

$139.46(17)$

$-40.5(2)$

$-1.1(2)$

$-179.88(15)$

$1.1(3)$

$-0.4(3)$

$-0.3(3)$

$0.2(3)$

0.5 (3)

179.25 (15)

Hydrogen-bond geometry $\left(A,{ }^{\circ}\right)$

$\mathrm{Cg}$ is the centroid of the $\mathrm{C} 4-\mathrm{C} 9$ ring.

\begin{tabular}{lllll}
\hline$D-\mathrm{H} \cdots A$ & $D-\mathrm{H}$ & $\mathrm{H} \cdots A$ & $D \cdots A$ & $D-\mathrm{H}^{\cdots} A$ \\
\hline $\mathrm{N} 1-\mathrm{H} 1 N \cdots \mathrm{O} 1^{\mathrm{i}}$ & $0.85(2)$ & $2.01(2)$ & $2.844(2)$ & $168(2)$ \\
$\mathrm{N} 3-\mathrm{H} 3 N \cdots \mathrm{C} 1^{\mathrm{ii}}$ & $0.97(2)$ & $2.12(2)$ & $3.065(1)$ & $167(2)$ \\
$\mathrm{C} 10-\mathrm{H} 10 B^{\cdots}{ }^{\mathrm{O}} 1^{\mathrm{iii}}$ & 0.99 & 2.40 & $3.151(2)$ & 132 \\
$\mathrm{C} 11-\mathrm{H} 11 A \cdots \mathrm{C} 1^{\mathrm{iii}}$ & 0.99 & 2.80 & $3.668(2)$ & 147
\end{tabular}


supporting information

$\begin{array}{lllll}\mathrm{C} 12-\mathrm{H} 12 A \cdots \mathrm{Cl} 1 & 0.99 & 2.81 & 3.520(2) & 129 \\ \mathrm{C} 12-\mathrm{H} 12 B \cdots \mathrm{O} 1^{\mathrm{i}} & 0.99 & 2.26 & 3.123(2) & 144 \\ \mathrm{C} 13-\mathrm{H} 13 A \cdots \mathrm{N} 1 & 0.99 & 2.53 & 3.138(2) & 120 \\ \mathrm{C} 14-\mathrm{H} 14 A \cdots \mathrm{C} 11^{\mathrm{iii}} & 0.99 & 2.71 & 3.585(2) & 147 \\ \mathrm{C} 21-\mathrm{H} 21 \cdots \mathrm{Cl} 1 & 0.95 & 2.83 & 3.757(2) & 165 \\ \mathrm{C} 18-\mathrm{H} 18 \cdots \mathrm{Cg}^{\mathrm{ii}} & 0.95 & 2.83 & 3.487(2) & 127\end{array}$

Symmetry codes: (i) $-x,-y+1,-z$; (ii) $-x+1,-y+2,-z$; (iii) $x+1, y, z$. 